Artículos

\title{
El logo, el branding y su influencia en la marca. Caso de estudio: el logosímbolo de Ecopetrol 2003*
}

\author{
Logo, Branding and their Influence on the Brand. A Study Case: The Logo-Symbol of Ecopetrol 2003 \\ O logo, o branding e sua influência na marca. Caso de estudo: o logosímbolo de Ecopetrol 2003
}

\author{
Maria Margarita Gutiérrez Gutiérrez ${ }^{\text {a }}$ \\ Corporación Universitaria UNITEC, Colombia \\ margaritaggutierrez@gmail.com \\ ORCID: http://orcid.org/0000-0002-5084-4995
}

DOI: https://doi.org/10.11144/Javeriana.syp38-75.lbim

Fecha de recepción: 31 Agosto 2018

Fecha de aprobación: 16 Febrero 2019

Fecha de publicación: 15 Noviembre 2019

\section{Resumen:}

Este artículo tiene dos objetivos generales: primero, analizar la identidad visual a partir de los cinco logosímbolos que desde 1951 ha tenido la Empresa Colombiana de Petróleos (Ecopetrol), con base en el análisis del signo, el ícono, el logotipo y el isotipo; y segundo, establecer el impacto del último logosímbolo sobre la percepción del consumidor, su aceptación y reconocimiento de las actividades, productos y responsabilidad socio-empresarial y ambiental de Ecopetrol, haciendo énfasis en el branding y su influencia en la marca. Para la consecución de estos objetivos, se diseñó una investigación cualitativa y cuantitativa, apoyada en el marco conceptual del marketing corporativo, a partir de lo cual se realiza esta reflexión.

Palabras clave: identidad visual, logosímbolos, branding, marketing corporativo, Ecopetrol.

\section{Abstract:}

This article has two general objectives: firstly, to analyze the visual identity in the five logo-symbols used by the Colombian oil company (Ecopetrol) since 1953, based on the analysis of the sign, the icon, the logo and the isotype. Secondly, to determine the impact of the last logo-symbol on the consumer's perception, the acceptance and recognition of the activities, products and socialcorporate and environmental responsibility of the company Ecopetrol. Emphasis is put on the branding and how it influences the brand. To attain these objectives, a qualitative and quantitative research was designed and supported by the conceptual framework of the corporate marketing. This reflection is developed as a part of this research.

Keywords: visual identity, logo-symbols, branding, corporate marketing, Ecopetrol.

\section{Resumo:}

Este artigo tem dois objetivos gerais: primeiro, analisar a identidade visual a partir dos cinco logo-símbolos que possui a Empresa Colombiana de Petróleos (Ecopetrol) desde 1951, com base na análise do signo, do ícone, do logotipo e do isotipo; e segundo, estabelecer o impacto do mais recente logosímbolo sobre a percepção do consumidor, seu aceite e reconhecimento das atividades, produtos e responsabilidade socioempresarial e ambiental de Ecopetrol, fazendo ênfase no branding e sua influência na marca. Para atingir esses objetivos, foi realizada uma pesquisa qualitativa e quantitativa, baseada no quadro conceitual do marketing corporativo, do qual esta reflexão foi realizada.

Palavras-chave: identidade visual, logosímbolos, branding, marketing corporativo, Ecopetrol.

Los contenidos de la comunicación corporativa, siendo de indole diversa, tienen siempre como protagonista a la propia empresa. Podríase decir que, de uno u otro modo, tiene un marcado acento de identidad, que hace posible la percepción integra de la globalidad de la empresa.

\section{Fuente: Ventura i Boleda}

Notas de autor: 


\section{Introducción}

Si bien en un principio el concepto del consumo estuvo vinculado a la figura del hombre primitivo en función de la consecución de alimento, vestido y abrigo, como tarea fundamental para el aseguramiento de su supervivencia, la sociedad de hoy va más allá del consumo biológico a uno enmarcado en un universo simbólico que resulta indispensable en la construcción de la representación del individuo en la vida cotidiana, e inscribe al ser social en las esferas individuales, públicas, sociales, políticas, económicas y culturales, y en el paradigma ${ }^{1}$ ambiental vigente en estos tiempos de crisis. Por lo anterior, ahora se habla del branding sustentable/sostenible convertido en "símbolo", no solo para la entrada de productos o servicios a los mercados, sino también como la manera de acceder a otros niveles perceptivos y de conocimiento, lo que permite una verdadera transformación de la marca, de los consumidores y de los sistemas involucrados, que impacte la parte psicológica-social, medioambiental y sustentable de la marca. Por ello, el mundo de la publicidad le ha impuesto un ritmo vertiginoso a la espiral de la propagación de elementos culturales, en la cual las marcas son un "objeto" que tiene profundas imbricaciones con los diferentes grupos sociales, lo que hace evidente que con la publicidad se crean fenómenos en los cuales salen a flote subculturas de consumo, como lo dice Lury (2004).

El término brand tiene su origen en la raíz escandinava o germánica que hacía referencia a "marcar con fuego", del que deriva branding, cuyo significado era "marcar al fuego". En sentido figurado, puede ser aplicado para hablar de los atributos de un producto que dejan impresión duradera en el consumidor por medio de la publicidad o la propaganda. Históricamente, el branding se inicia con la Revolución Industrial, cuando las corporaciones crearon las marcas como una manera de incrementar sus ventas en zonas alejadas del lugar inmediato de producción (Healey, 2008). No es casualidad que los primeros artículos que ostentó una marca fueran las bebidas alcohólicas para exportar. En la práctica, el branding se apoya en cinco elementos: posicionamiento, historia, diseño, precio y relación con el consumidor. Aunado a este concepto está el de brand equity, que, enfocado desde la perspectiva del cliente, y según Forero y Duque, la mejor definición es la de la American Marketing Association: "el valor de la marca se basa en la actitud de los consumidores sobre los atributos positivos de la marca y las consecuencias favorables del uso de la marca” (Forero y Duque, 2014, p. 161). En esencia, el cliente permite conocer el valor actual que los consumidores le dan a una marca, lo cual se denomina "valor marcario" o "capital de la marca", y es el conjunto de cualidades o responsabilidades asociadas con el nombre, símbolos o logos, lo que determina el valor que un producto o servicio proporciona a una compañía o a un cliente de esta; lo que resulta en su valor financiero, uso económico y fortaleza en el mercado (Aaker, 1996). Respecto al consumidor, lo anterior incide en la percepción de superioridad que tiene de la marca, la cual es medible a partir de indicadores para cada categoría -imagen, conciencia, posicionamiento, familiaridad, fidelidad, satisfacción, recomendación, conexión emocional, relevancia etc.-; y que en términos de marketing puede traducirse en los siguientes beneficios: (1) percepciones mejoradas del desempeño del producto, (2) mayor lealtad del cliente, (3) menor vulnerabilidad a las acciones competitivas del mercado y a las crisis económicas, (4) mayores márgenes; (5) mayor elasticidad de la demanda frente a la disminución de precios, y mayor inelasticidad como respuesta de los clientes a los incrementos de precios; (6) aumento de la eficacia en las estrategias de comunicación del marketing, y (7) licencias adicionales y mayores oportunidades de extensión de marca.

Mientras que en los años ochenta los ambientes comerciales estuvieron dominados por una demanda en el diseño de marcas, los noventa vivieron la evolución del branding. Al respecto, Silva (2011) expresa que "todo puede tener una marca, ya que constituye una promesa de satisfacción; puede ser un signo o una metáfora que se convierte en un acontecimiento para quienes lo experimentan" (p. 43). 
En su nivel más básico, la identidad gráfica está relacionada con el desarrollo de un sistema que engloba el estilo visual de la entidad a la que representa, y en términos de diseño le provee de identidad distintiva, reconocible, diferenciada y unificada. Se entiende que el diseño de la identidad -ya sea personal, empresarial, organizacional, corporativa o institucional- se ha centrado en el diseño de logotipos -o identificador de una empresa-, que es lo que la representa; sin embargo, este no está inscrito como una pieza publicitaria o una comunicación publicitaria directa, sino como un recurso gráfico que busca generar reconocimiento y recordación en la mente del público, así como lograr la identificación del anunciante -branding-, el cual debe entenderse como el acto de marcar o buscar identificar algo como propio (Martínez, 2011). De ahí que entre 1950 y 1970, la aparición del logo fue creciendo sustancialmente, pasando de estar en la etiqueta a robarse la atención de la mayoría de los objetos, pues "gradualmente, pasó de ser una afectación ostentosa para convertirse en un accesorio esencial de la moda" (Klein, 2001, p. 56). Gracias a que las imágenes dotan de significado y posibilitan el conocimiento y aprendizaje de una realidad (la empresa, la marca), estas cumplen para el sujeto (por ejemplo, consumidor o inversionista) una función de simplificación de los procesos de obtención de información y búsqueda de oferta, que les facilita decisiones de consumo (Poiesz, 1988).

Por ello, destacados exponentes del diseño gráfico, como Olins (2004), se han preocupado porque el sistema de identidad de las grandes empresas se extienda a todas sus manifestaciones visuales, hasta el punto de convertirse en sinónimo de su personalidad. De ahí la importancia de las nociones inmateriales de la imagen (la impresión intangible que proyecta la empresa hacia el exterior). Así, no es raro que en los últimos años se haya generado una nueva área de estudio: el "marketing corporativo", fruto del desarrollo de la investigación académica en torno a conceptos interrelacionados, como identidad, imagen, personalidad, branding o comunicación, referidos a la organización en su totalidad y a la empresa en su conjunto (Balmer, 2008). Por ello, la "identidad visual" hace referencia a la representación gráfica de la "identidad corporativa" en forma de símbolos y señales, como marcas, logotipos, logosímbolos, decoración, estilo corporativo, vestuario del personal, etc. (Van Riel, 1995; Chajet y Shachtman, 1998; Kotler y Armstrong, 2008). Por ello, Christensen y Askegaard (2001) afirman que es la noción más empleada en el área de marketing, y quizás la que sufre mayor ambigüedad. Sin embargo, al revisar y delimitar el concepto, Currás (2010) concluye que hay conceptualizaciones que apuntan a considerarla como el ethos de la empresa y cómo este se representa y se manifiesta, mientras que otros autores lo ciñen exclusivamente a los "modos" que tiene la organización de representarse a sí misma. La indagación bibliográfica permite concluir que "identidad e imagen corporativas" están interrelacionadas, ya que las impresiones y percepciones de los públicos (imagen) se construyen sobre la comunicación de la organización (identidad); no obstante, es difícil especificar cuál es el impacto del logo después de llegar a ser conocido por la sociedad, ya que la marca - representada por su ícono o logo, y su intervención en el mercado o en la sociedad en general- puede generar consecuencias positivas o negativas. En esta reflexión llevada a cabo a partir de un caso de estudio enfocado en Ecopetrol, se analiza la evolución histórica de sus logosímbolos -sobre todo el de 2003- y se dilucida su influencia en el branding y en la marca, a partir de su análisis gráfico y semiótico, con el fin de constatar si lo que este representa y comunica corresponde con lo que ocurre respecto al cumplimiento de las obligaciones y responsabilidades, y se esclarece si se está ante un típico caso de greenwashing.

\section{La historia de Ecopetrol}

En 1950, Suramérica tenía parte importante de la producción global de hidrocarburos, al ofertar el 17\% de esta (3.756.000 bd [barriles diarios]); sin embargo, esto trajo consigo debates y conflictos políticos y sociales en países como Bolivia, Chile y México. Una de las principales discusiones fue la del carácter privado o público de la explotación, la cual estuvo centrada en si debían mantenerse los contratos de concesión entre el Estado y las compañías privadas, si la actividad podría ser desarrollada en conjunto por los sectores público y privado, 
o si definitivamente el Estado debía encargarse de la totalidad de las etapas de la cadena productiva. De ahí que las expropiaciones y las restricciones o exclusiones de las empresas foráneas empezaran a ser recurrentes, por lo cual el debate se intensificó y se propagó por varios países de la región. En cada país, la discusión y los procesos institucionales tuvieron diferencias y similitudes, y Colombia no fue la excepción, por lo que en la década de los cuarenta, se empezó a discutir la posibilidad de crear una compañía petrolera de carácter estatal. Bajo la presión popular, se quería que las decisiones de la política a adoptar terminaran protegiendo los intereses nacionales, y ese fue el caso de la actividad petrolera (Philip, 1982).

Los estudios sobre la historia petrolera de Colombia no han sido muchos (Bucheli, 2010). La puesta en marcha de Ecopetrol como compañía estatal fue producto de la presión popular, y tuvo como origen la huelga de enero de 1948, la cual fue consecuencia del despido de 500 trabajadores por parte de la Tropical Oil Co (De La Pedraja, 1993). La exigencia de los trabajadores incidió en que el presidente Ospina Pérez constituyera el Consejo Nacional de Petróleos y en que se empezara a discutir esta idea. Al mismo tiempo, tuvo lugar la reversión de la Concesión de Roberto de Mares (Villegas, 1976; Montaña-Cuéllar, 1977; Vega, Núñez y Pereira, 2009); y en 1951, se funda la Empresa Colombiana de Petróleos (Ecopetrol), lo que se convirtió en una buena señal de soberanía que en algo alivió el descontento social que imperaba. En la tabla 1 se consignan los principales sucesos de la extensa historia de Ecopetrol. Se destaca que en 2003, cambia su naturaleza jurídica, convirtiéndose en una sociedad pública por acciones (Decreto 1760, 2003), lo que le dio el nombre de Ecopetrol S.A. Este cambio se realizó con el fin de internacionalizarla, y de hacerla más competitiva y financieramente más fuerte.

TABLA 1

Línea histórica de Ecopetrol

\begin{tabular}{ll}
\hline Año & Suceso \\
\hline 1951 & $\begin{array}{l}\text { Nace la empresa Colombiana de Petróleos (Ecopetrol). Reversión al Estado colombiano de la } \\
\text { Concesión De Mares. }\end{array}$ \\
\hline 1961 & Asume el manejo de la refinería de Barrancabermeja \\
\hline 1970 & Ratifica su naturaleza de empresa industrial y comercial del Estado \\
\hline 1974 & Compra la refinería de Cartagena \\
\hline 1983 & Descubre el campo de Caño Limón (Arauca) en asocio con la OXY \\
\hline 1986 & Colombia vuelve a ser un país exportador de petróleo \\
\hline 1989 & Descubre el campo Cusiana (Casanare) en asocio con la BP \\
\hline 1993 & Descubre el campo Cupiagua (Casanare) \\
\hline 2003 & $\begin{array}{l}\text { Se convierte en sociedad pública por acciones (Decreto 1760) y se denomina Ecopetrol S.A. } \\
\text { Renueva su marca e identidad corporativa: se cambia el logo del tunjo por el de la iguana. }\end{array}$ \\
\hline 2007 & $\begin{array}{l}\text { Realiza el primer proceso de recapitalización. 482941 colombianos adquieren acciones. El Estado } \\
\text { conserva el 89,9\% de las acciones. }\end{array}$ \\
\hline 2008 & Compra Propileo y constituye el Grupo Empresarial Ecopetrol \\
\hline $2009 / 10$ & $\begin{array}{l}\text { Compra Hocol, Reficar y adquiere participación en Savia (Perú), Ocensa y BP en Colombia. } \\
\text { Amplia su presencia exploratoria en Estados Unidos, Brasil y Perú, e ingresa a los mercados de } \\
\text { Asia y Africa. Ingresa a la Bolsa de Valores de New York, Toronto y Lima. }\end{array}$ \\
\hline 2011 & $\begin{array}{l}\text { Celebra su sexagésimo aniversario. Realiza la segunda ronda de emisiones de acciones. Ingresa al } \\
\text { indice de Indicadores de Sostenibilidad Dow Jones. }\end{array}$ \\
\hline 2012 & $\begin{array}{l}\text { Crea Cenit Transporte y Logística de Hidrocarburos S.A., filial 100\% de propiedad de } \\
\text { Ecopetrol. }\end{array}$ \\
\hline
\end{tabular}




\section{¿Qué es y para qué sirve un logo?}

¿Cuál es el significado profundo que expresan las marcas, logotipos, isotipos, tipografías, avisos, etc.? ¿Qué aspectos inconscientes se expresan mediante la imagen y el color? ¿Cómo diseñar desde un logotipo hasta un sistema completo de identificación visual, teniendo en cuenta el sentido connotado que encierran las líneas, las letras, las formas y las tonalidades? Figurativamente, el logotipo es la "firma de una marca, símbolo de garantía y responsabilidad y como tal es único e irrepetible” (Pol, 2005, p. 64). Igual que sucede con la firma y rúbrica de una persona, los distintos rasgos gráficos de los logos e isotipos proyectan determinada personalidad y conforman la identidad visual de la marca, la cual, como firma del producto, comunica su personalidad mediante las formas, los colores y los símbolos condensados en el logotipo.

Actualmente existen marcas globales, por lo cual las necesidades comunicacionales han cambiado. La exigencia de desarrollar respuestas destinadas a mercados amplios y consumidores heterogéneos, tanto cultural como geográficamente, obligan a concebir logotipos e isotipos globales. Cobra importancia, entonces, el componente visual, en el cual radica la posibilidad de universalizar el sentido, ya que la dimensión verbal de la marca no siempre será decodificada "por todos y de la misma manera”. A su vez, las marcas globales se deben a la existencia de ciertos arquetipos universales cromáticos, los cuales constituyen el lenguaje común que las vivifica $^{2}$.

Si el símbolo es un puente o entidad intermedia entre lo oculto y lo manifiesto, lo inconsciente y lo consciente, lo connotado y lo denotado, lo reprimido y lo expresado; entonces la marca-como sistema de símbolos en el espacio gráfico- es la expresión de contenidos profundos que se proyectan hacia la superficie. Para interpretar el lenguaje de los símbolos gráficos y la personalidad que subyace en ellos, es imprescindible el análisis de las estructuras gráficas - letras, trazos, espacios en blanco, etc.- desde el simbolismo del espacio y el estudio de los arquetipos universales (formales, espaciales y cromáticos), trasladados al campo de la Comunicación Visual, el Diseño Gráfico y la Publicidad. De ahí que la identidad corporativa sea parte fundamental de la comunicación empresarial, pues se asocia con su imagen y la convierte en el conjunto total de la cultura interna de la empresa. Por ello, cuando se habla de elementos publicitarios relevantes, el logo es quizás uno de los protagonistas con mayor relevancia. Este debe ser entendido, según Caldevilla (2010), como "la manera de expresar visualmente la denominación de la entidad, su forma visual" (p. 194), y tiene como objetivo exaltar y buscar la manera de identificar un conjunto de elementos que cualquier corporación posee, los cuales necesita comunicar, mostrar y perpetuar de manera concisa y clara ante una fracción objetivo de la sociedad. Por ello, existen logos de todo tipo, como los tipográficos -o compuestos únicamente por letras- o aquellos que solo poseen isotipos construidos a partir de una imagen (Pol, 2005; Caldevilla, 2010).

El desarrollo gráfico de un logo posee varias etapas, en las cuales se debe definir si la representación visual será un isotipo, un logotipo o un logosímbolo. Este último contiene una representación gráfica y un conjunto tipográfico. Tomar esta decisión también depende del momento en el cual se encuentra la empresa; en el caso de los isotipos, son usualmente utilizados cuando el reconocimiento social es muy alto y el símbolo por si solo logra evocar la empresa, mientras que los logos tipográficos y los logosímbolos son utilizados cuando hay más información que presentar o la entidad es muy reciente. Para poder lograr un identificador adecuado, que exprese de manera adecuada lo que la empresa necesita, se requiere información sobre a qué se dedica la empresa y cuál es el diferencial que se quiere resaltar, proceso que se conoce como briefing. El brief es un documento que se presenta al cliente, o a quienes desarrollarán el concepto visual de la empresa, en el cual se consolida la información de la empresa, su misión, visión, oficio, intereses, metas y los deseos del cliente respecto a la representación gráfica a realizar. Al tener claro el contenido que debe poseer la identidad visual del logo, se procede a buscar una forma que sintetice la información, teniendo como premisas que debe ser llamativa y que debe lograr recordación en quienes estén expuestos a ella. Para esto, se deben tener en cuenta la familia tipográfica, el color, el tamaño y el equilibrio. 
Publicitariamente, un logo se convierte en el elemento que recoge la ideología y actos que una empresa posee o ejecuta, y su puesta visual externa, la de sus productos y servicios, podría asimilarse a "una esponja", pues absorbe, tanto lo positivo como lo negativo. Así, la representación sería "la cara, la imagen” de la(s) empresa(s), en términos de presentación. De ahí que se entienda que toda la carga publicitaria y social está concentrada en el logo, ya que, además, entrega a la sociedad de manera subliminal la responsabilidad social que se asume, respecto a su potencial mercado o clientes. Aun teniendo en cuenta que el logotipo debe ser "único e irrepetible", a lo largo de su historia, una marca o empresa puede haber tenido varios logos. Ese el caso de Ecopetrol, que ha modificado su imagen corporativa en cinco ocasiones.

En este sentido, y como primer elemento de análisis previo a interpretar cada uno de los logos, es pertinente establecer el significado y el sentido histórico otorgado a los colores en los diversos distintivos nacionales, ya que es un rasgo cultural aprehendido cuando de verlos se trata. De hecho, Ecopetrol ha recurrido a estos imaginarios. Es así como el pabellón de Colombia tiene su origen en la composición tricolor creada en 1801 por el general venezolano, Francisco de Miranda, quien describió en su diario militar el uso de los colores primarios en la bandera para representar a las naciones latinoamericanas -que en aquel entonces estaban en proceso de independencia- y que terminó representando a la Gran Colombia (Castrillón, 1961). Los colores fueron adoptados de forma sucesiva, primero por el Congreso de la Gran Colombia, el 17 de diciembre de 1819; y después, por el Congreso de la República de la Nueva Granada, el 9 de mayo de 1834. La disposición actual de estos fue adoptada de forma definitiva el 26 de noviembre de 1861 (Castrillón, 1961). La primera descripción de los colores del pabellón nacional, así como la interpretación de su significado, se le atribuye al patriota Francisco Antonio Zea, quien, durante su discurso en el Congreso de Angostura de 1819 (congreso en el cual nació la Gran Colombia), declaró:

nuestro pabellón nacional, símbolo de las libertades públicas, de la América redimida, debe tener tres franjas de distintos colores: sea la primera amarilla, para significar a los pueblos que queremos y amamos la federación; la segunda azul, color de los mares, para demostrar a los déspotas de España, qué nos separa de su yugo ominoso: la inmensidad del océano, y la tercera roja, con el fin de hacerles entender a los tiranos que antes de aceptar la esclavitud que nos han impuesto por tres siglos, queremos ahogarlos en nuestra propia sangre, jurándoles guerra a muerte en nombre de la humanidad; en el centro del escudo pondremos la imagen de nuestro cóndor andino, a imitación de los romanos que colocaron en sus banderas las famosas águilas que conquistaron el mundo. (Castrillón, 1961 p. 65)

\section{Recorrido histórico de los logosímbolos de Ecopetrol (1951-2003)}

El primer logosímbolo data de 1951, y fue reflejo de las circunstancias sociales, económicas y políticas que imperaban en Latinoamérica y el Caribe. En el caso colombiano, obedeció a las exacerbadas protestas sociales sobre la propiedad de los recursos naturales, en este caso, las relacionadas con las disputas ocasionadas por el destino de las riquezas petroleras, aprovechadas políticamente por los partidos conservador y liberal, y la izquierda representada en los sindicatos (figura 1). 


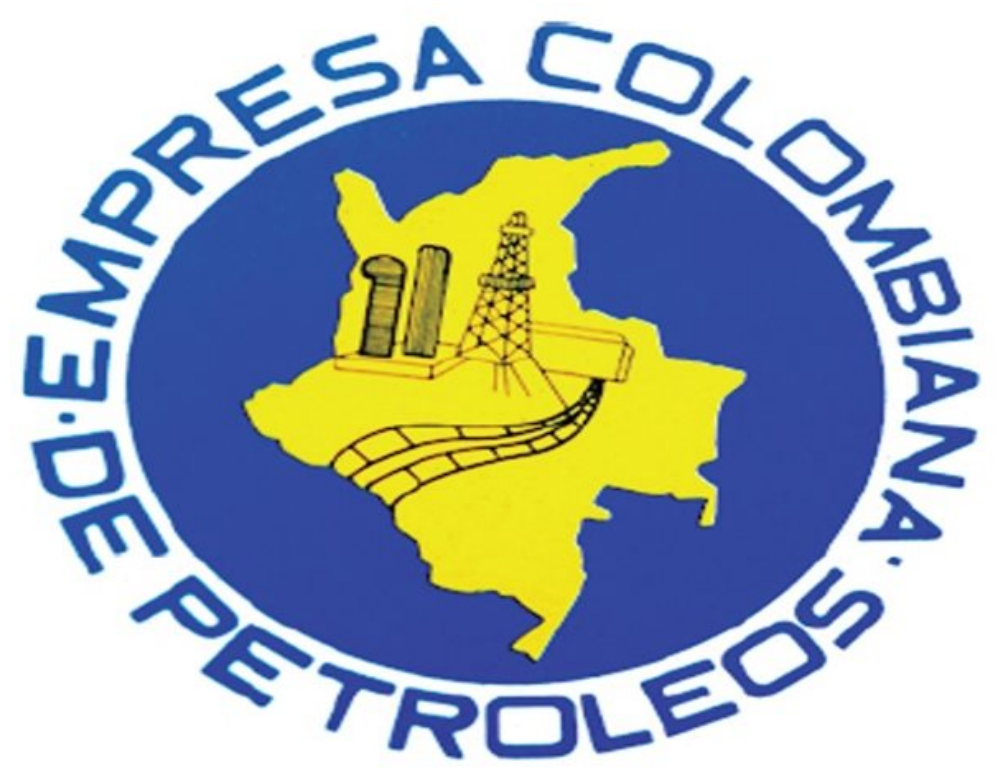

FIGURA 1

Primer logosímbolo de Ecopetrol (1951)

Fuente: Ecopetrol (2011, p. 351)

Así mismo, respondió a la necesidad de dar a entender que la entidad creada, encargada del manejo y administración de los hidrocarburos, defendería los intereses nacionales ${ }^{3}$. El logosímbolo identifica a la empresa como nacional, independiente de agentes foráneos y como defensora de los recursos petroleros, de ahí, que se incluyan el mapa de Colombia, la imagen de la torre petrolera, las refinerías y los caminos que allí conducen. Visualmente, genera la sensación de que la actividad está inmersa en todo el país, sin distinción de regiones; y de manera externa, es visible que la empresa aglutina al país y cobija todas las actividades petroleras. Es un logosímbolo racional que apeló a lo que la sociedad quería ver representado: una empresa de y para los colombianos. Desde el punto de vista del color, se recurrió al azul, que representa los mares, que siempre hemos asimilado a riqueza; y al amarillo, que representa la unión.

En la segunda versión del logosímbolo (1963) (figura 2), las ideas de pertenencia y sentimiento nacionalista se reafirman con la mención completa del nombre de la empresa. Se instaura el acrónimo de la empresa, Ecopetrol, y se acude a un símbolo que reúne tanto la riqueza del "oro negro", como las riquezas ancestrales nativas, representadas e inmersas en un Tunjo precolombino Muisca, inscrito en un hexágono, el cual a su vez representa la molécula de carbono -elemento principal del petróleo. Valga decir que para los orfebres muiscas, el arte tenía doble significado: expresión estética y simbolismo religioso, aunque su terminación en la imagen sea descuidada y muy geométrica (Constela-Umaña, 1995). 


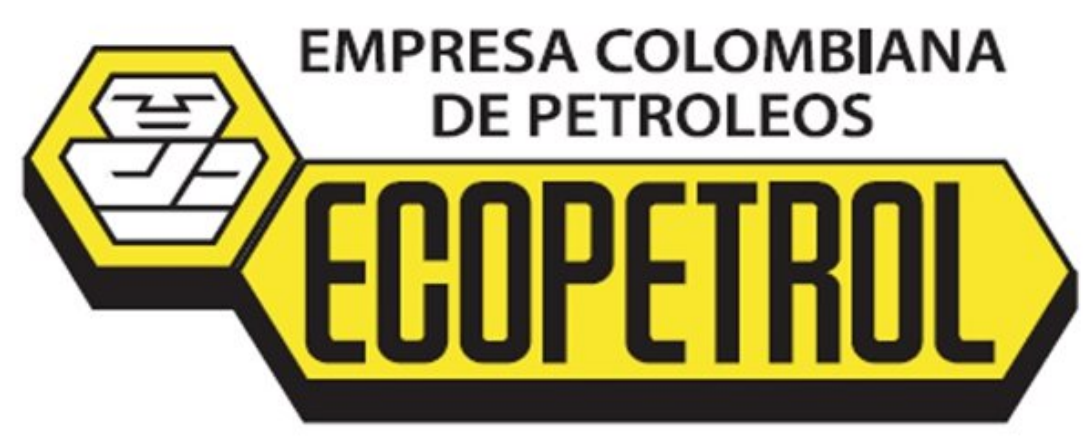

FIGURA 2

Segundo logosímbolo de Ecopetrol (1963)

Fuente: Ecopetrol (2011, p. 351)

A diferencia del primer logosímbolo, en el segundo, los colores cambian: ahora predomina el amarillo, que sirve como expresión de unidad, pero también se mantiene el color negro -oro negro. Se utilizó durante 20 años y fue cambiado por el presentado en la figura 3, el cual fue diseñado por Benjamín Villegas (Ballestas, 2009).

El tercero (figura 3) se presentó en 1983, y viene siendo la abstracción del tunjo precolombino planteado en el logo de 1963. Se siguió apelando al concepto de la pertenencia nacional de la empresa, evocando la creencia en la identidad y el arraigo cifrado en la cultura precolombina, respecto a la cual se afirmaba que "los indígenas se bañaban con barro y petróleo y, que parece estarlo representando la figura” (Ballestas, 2009, p. 207).

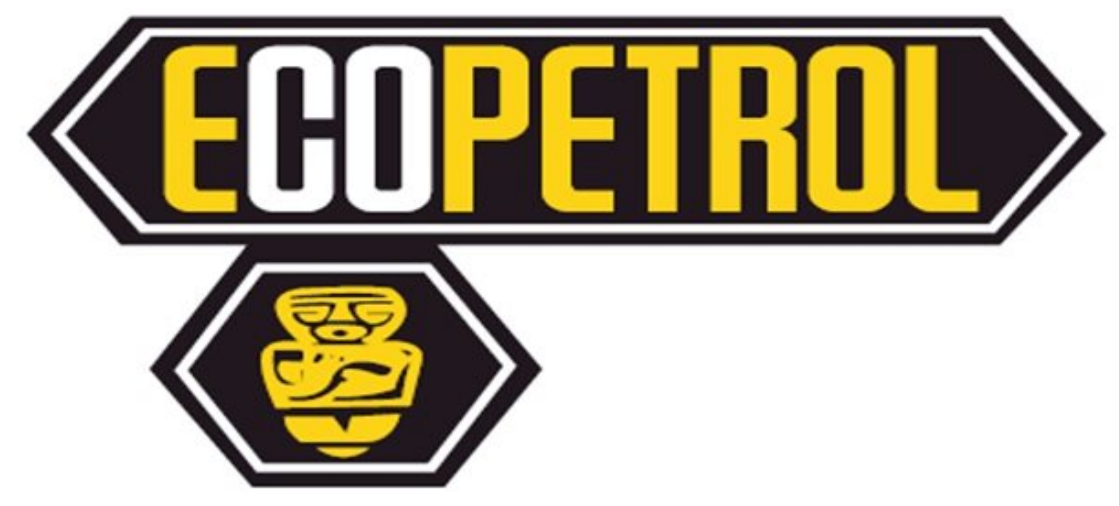

FIGURA 3

Tercer logosímbolo de Ecopetrol (1983)

Fuente: Ecopetrol (2011, p. 351)

De igual manera, induce a pensar que la empresa hace aprovechamiento racional de los recursos naturales, en coherencia con la cultura indígena que así lo hacía. La entidad seguía siendo $100 \%$ propiedad del Estado. Se elimina el nombre de la empresa y se acude al acrónimo -Ecopetrol-, con el objetivo de probar la recordación. A diferencia del segundo logosímbolo, los colores cambian; en el segundo predominaba el amarillo, y en el tercero, el negro - oro negro-. Con relación al tunjo, se modifica su composición, posición y color, pero en términos generales, se conserva la identidad corporativa.

El cuarto logosímbolo (figura 4) es muy similar al tercero, una expresión racional y sistemática basada en términos conceptuales lógicos. A pela a generar un top of mind, que hace referencia a la recordación de la marca, mas no a su preferencia (Hoyos, 2013). El acrónimo de la empresa permanece, al igual que el nombre completo -Empresa Colombiana de Petróleos. A diferencia del tercer logosímbolo, no se resalta el componente CO, 
como si fuera abreviación de Colombia, en color distinto, pues la empresa seguía siendo 100\% nacional. Este tercero estuvo vigente durante 14 años.

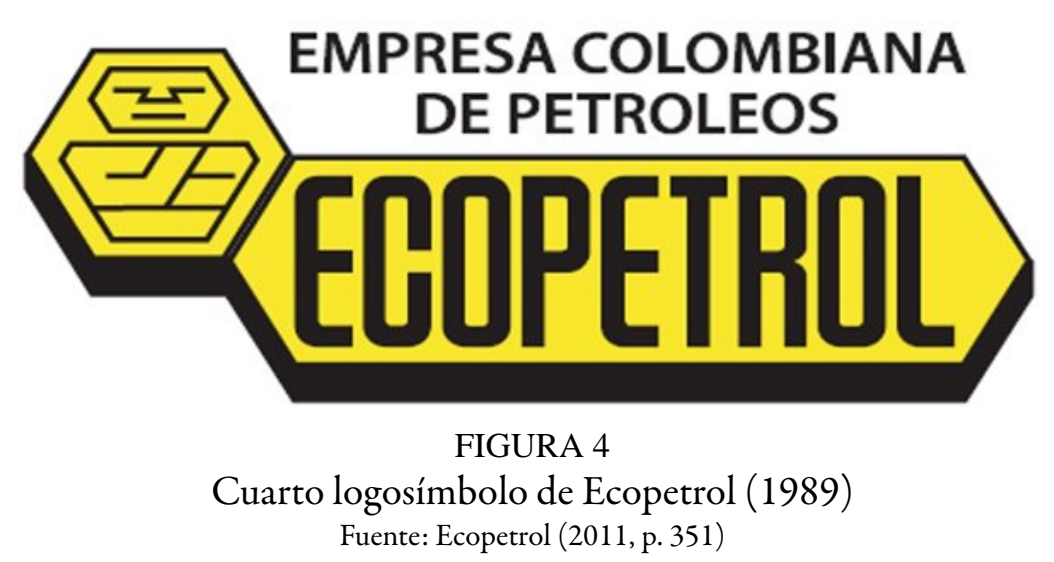

Sin embargo, la empresa se transformaría en el 2003 al denominarse Ecopetrol S.A., convirtiéndose así en una sociedad de economía mixta, de carácter comercial, organizada bajo la forma de sociedad anónima, del orden nacional, vinculada al Ministerio de Minas y Energía, y liberada de las funciones del Estado como administrador del recurso petrolero -que pasó a ser campo de acción de la Agencia Nacional de Hidrocarburos (ANH)-, lo que le generó autonomía, y mayor capacidad empresarial y comercial. Lo anterior incidió en que se convirtiera en la empresa más grande del país, con una utilidad neta para el año 2017 de \$ 6,6 billones. Por su tamaño, pertenece al grupo de las 40 petroleras más grandes del mundo y una de las cuatro principales de Latinoamérica (Corcho Tróchez, 2013).

El logosímbolo de la figura 5 es la última imagen corporativa de Ecopetrol S.A, la cual abandona la orientación nacionalista para dar paso a una representación con elevados tonos ambientalistas, utilizando diversos tonos de verde que corresponden con la moda ecologista imperante y con las exigencias sociales respecto al medioambiente. A continuación, este será objeto de análisis de carácter técnico como simbólico referidos al logotipo, el isotipo y el logosímbolo como conjunto.

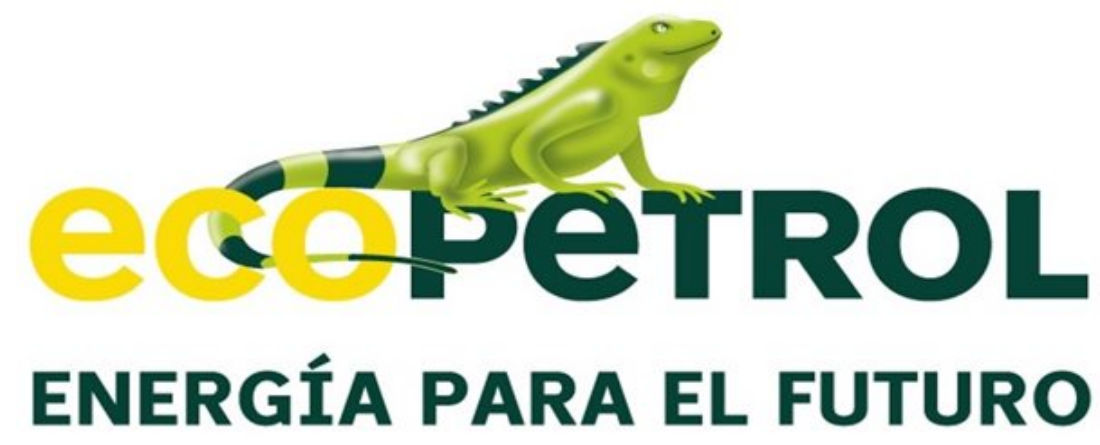

FIGURA 5

Quinto logosímbolo de Ecopetrol (2003)

Fuente: Ecopetrol (2011, p. 351) 


\section{Análisis gráfico}

\section{El logotipo}

El logotipo, conformado por letras o tipografía, posee como destacables dos elementos: la abreviación del nombre de la empresa "Ecopetrol" y el slogan "energía para el futuro". En términos técnicos, el logo contiene un juego de tipografía entre altas y bajas. Las dos vocales "e" están en minúsculas; y las demás letras, en mayúsculas (figura 6). Este elemento le da al logo la posibilidad de generar unidad, lo que lo hace más amigable. Este atributo también se maneja con la separación entre letras (kerning y tracking [interletreado]).

\section{ECOPETROL ENERGÍA PARA EL FUTURO \\ FIGURA 6 \\ Descomposición del logosímbolo (2003) Fuente: Ecopetrol (2011 p. 351)}

Los dos elementos tienen tipografía palo seco, lo cual en textos cortos brinda más flexibilidad, lo que genera sensación de frescura y tranquilidad, pero, sobre todo, de novedad y adaptabilidad (Zanon, 2007). La tipografía, como lo asegura el manual de identidad, está construida en minúsculas; sin embargo, la idea que genera es diferente; el juego y el cambio de letras altas a bajas brinda la misma sensación de novedad que se percibe con la creación de una nueva tipografía palo seco. El slogan está presente en letras altas sostenidas, lo que ayuda a hacer énfasis en la idea de renovación, y a cerrar con la idea de que lo que pretende la empresa: "energía para el futuro". Los dos elementos tipográficos están construidos a partir de una caja de texto, con simetría y equilibro, lo que le da al logotipo la posibilidad de estar en juego con la idea de novedad que brinda la tipografía, sin llegar a ser un logo que se torne juvenil o poco serio.

Otro elemento relevante tipográficamente es el manejo de los colores, cálidos y fríos. En la categoría de los cálidos está el amarillo, que resalta la palabra "ECO", la cual en los antiguos logos hizo alusión a "Empresa Colombiana de Petróleos", y ahora toma el protagonismo en términos ecológicos. El amarillo, según Ricupero (2007), emite sensaciones de juventud, novedad, serenidad y luz; es un color que llama a lo nuevo, y el autor lo define como "un color que empuja, hacia lo nuevo, lo moderno, el futuro" (p. 20). No obstante, lo más importante es que llama a la vida, justo aquello que Ecopetrol quiere resaltar con su cambio de imagen. La segunda parte de la palabra Ecopetrol, PETROL y el slogan "energía para el futuro", tienen un color frío, un tono verde oscuro alusivo al petróleo mismo, sin migrar a los tonos negros, manteniendo así una gama de color estable. El verde, que representa esperanza, tranquilidad y serenidad, también denota juventud, lo cual debe ser entendido como el deseo de unidad. El haber consultado públicamente la identidad visual permitió establecer una combinación de colores que generara la idea de equilibrio emocional -(1) vigoroso y fuerte y (2) con gran contraste-, lo que evidencia serenidad y confiabilidad, ante lo cual la combinación resultaba exitosa en términos estéticos y psicológicos. La concepción general del logosímbolo pretendía conectar a los colombianos al anunciar que asumía el reto de crear futuro, y como lo expresa textualmente la empresa que lo 
diseñó: "por supuesto coqueteamos con los temas ambientales y el compromiso con la sostenibilidad" (Mesa, 2013, p. 1).

\section{El Isotipo}

La iguana, el isotipo o "imagen-forma" que acompaña el logotipo, tiene representación caricaturesca de la especie, que en nada corresponde con el aspecto agresivo que posee en el medio natural: una especie anfibia, distribuida sobre todo en el Magdalena Medio, donde la empresa tiene presencia (figura 7). Según Ecopetrol (2011), la iguana representa la calidad medioambiental de los ecosistemas en donde ejerce sus actividades. Así mismo, es representativa y de mucho valor, bien como soporte alimentario o por el comercio de sus huevos para las comunidades locales - razones por las cuales está amenazada-, lo que refuerza su uso en el logosímbolo como mensaje subliminal de protección y conservación.

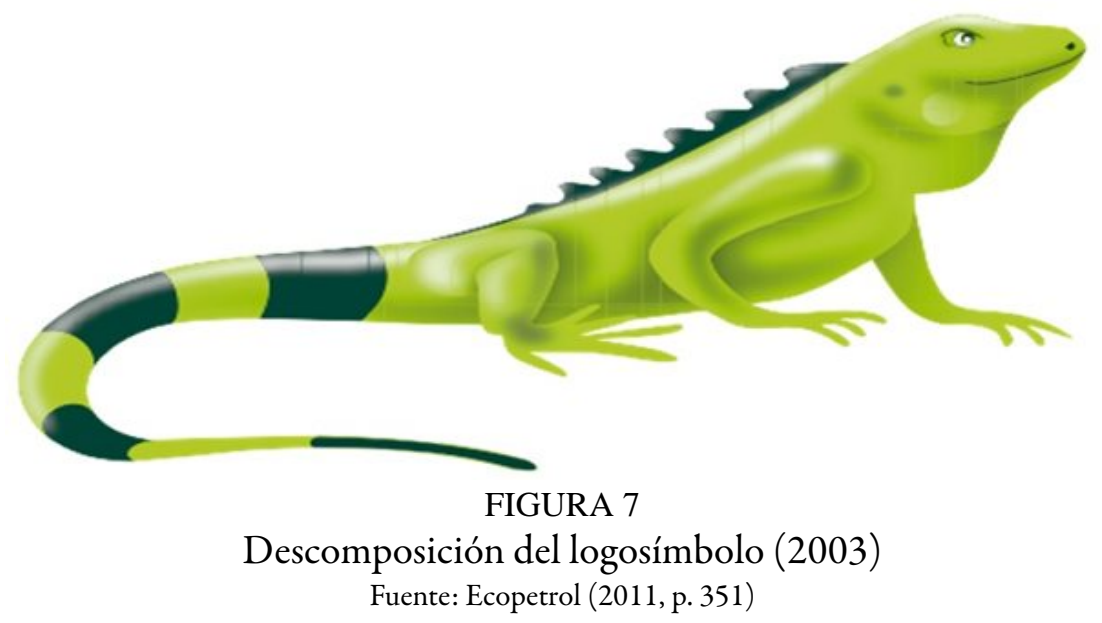

El resaltar con diferente color la palabra "ECO" es un nuevo recurso gráfico que ayuda a que se identifique a Ecopetrol S.A. como una empresa amigable con el medioambiente, ecológicamente respetuosa y coherente con lo que la sociedad ahora exige. Además, denota que se interesa por responder a las políticas del Estado que propende por la conservación y la recuperación de los ecosistemas y su biodiversidad.

En términos gráficos y técnicos, la iguana posee amplia gama de verdes, que, según la psicología del color, evoca esperanza, renovación, luminosidad, tranquilidad, armonía, prosperidad y ganancia (Ricupero, 2007). Además, denota la naturaleza en todo su contexto, concepto central del mensaje a interiorizar por quienes lo vean. Partiendo de que incluso lo feo, despreciado por ser la antítesis de lo bello, puede ser objeto de la reflexión estética -que a mediados del siglo XIX ocupó el lugar central en una obra de Rosenkranz-, se puede afirmar que la caricaturización del isotipo se hace llamativa, sin que llegue a ser la antítesis de la belleza establecida por Eco (2010); y como lo expresa Sánchez (1992), "se llama bello a un objeto que por su estructura formal, gracias a la cual se inscribe en ella cierto significado, produce un placer equilibrado o goce armonioso" (p. 185). La intención de caricaturizar la iguana era volverla más estética y agradable, la idea es que su trazo, similar al de un dibujo animado, amplifique el público objetivo o target, en tanto que es llamativo para los niños y retrotrae en los adultos el concepto de lo ecológico y respeto por la naturaleza. Es tal la apropiación lograda con el logosímbolo, que es común escuchar entre los trabajadores de la empresa que trabajan "en la iguana”. 


\section{El logosímbolo}

La iguana, como elemento central, luce cómoda, plácida y entrelazada con la empresa y sus actividades. Además, la dirección de la mirada y la diagonal de su columna vertebral son símbolos que emulan el tiempo y el progreso: el primero transcurre hacia la derecha y el segundo está representado con la flecha que apunta progresivamente hacia arriba o hacia adelante. Integralmente, el logosímbolo tiene características muy técnicas, las cuales desde la formalidad logran generar como resultado un aspecto de seriedad y movilidad, debido al tratamiento cromático y tipográfico. En el isotipo se encuentra que a partir de las características técnicas, como el trazo y el color, se logra desarrollar toda una simbología que genera un complemento armónico, estético y funcional. Aunque el logosímbolo sea complejo, tiene elementos técnicos que llevan al consumidor simbologías caracterizadas por lo siguiente. Primero, como se advierte, la iguana posa sobre el nombre de la empresa, su cola entrelaza la palabra "ECO” y sus patas parecen cómodas -que hace alusión a que la extracción petrolera brinda seguridad y acoge a la iguana, lo que genera una sensación de armonía entre esta y el entorno natural, ya que, aun estando amenazada como especie, parece estar protegida por las actividades de la empresa. Este elemento juega el mismo papel que el de las letras "e" en bajas, que genera unidad y muestra la capacidad del ambiente para estar en equilibrio con las acciones petroleras o viceversa. Segundo, la iguana mira hacia el frente con determinación, lo cual unifica el slogan puntualmente con la palabra futuro, y deja claro el mensaje que Ecopetrol y sus actividades con el medio ambiente son compatibles, ya que pueden ser amigables, no antagónicas, y garantizan un futuro certero y seguridad en todos los aspectos (figura 8).

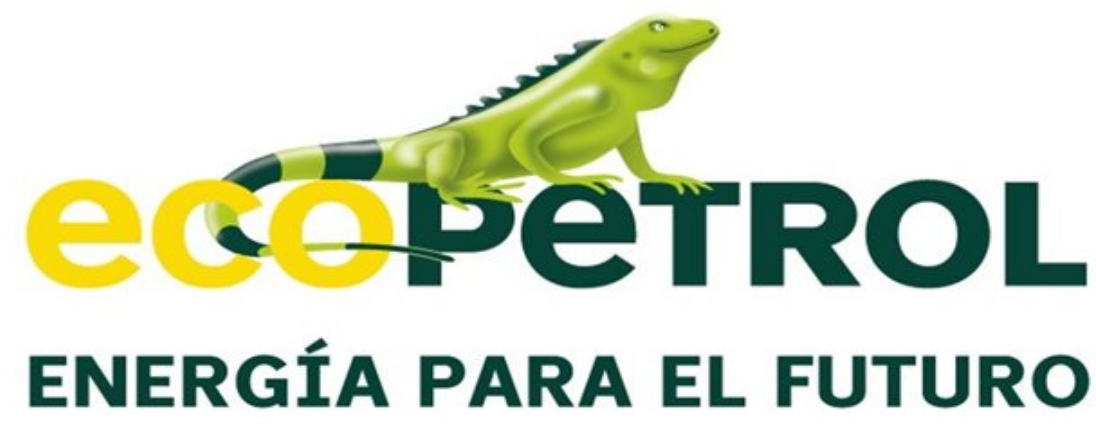

FIGURA 8

Descomposición del logosímbolo de Ecopetrol (2003)

Fuente: Ecopetrol (2011, p. 351)

En sus diversas comunicaciones, Ecopetrol S.A. resalta el poder del prefijo "ECO" al explicar que viene de oikos, que significa casa. Con esto se refiere al planeta tierra como nuestra casa. Una visión "ECO" contempla principios superiores y valores orientados a preservar la vida, vida que la iguana comunica con la maestría de lo simple, pero se sabe y está comprobado que en la práctica empresarial no tienen lugar. A su vez, captura las cosas realmente importantes para todos, y con el apoyo del eslogan afirma que la vida se perpetúa infinitamente. Respecto al significado otorgado a la iguana, el diseñador manifestó: "después de una década, la iguana se ha convertido en un ícono fascinante al que otorgamos nuevos sentidos que agudizan su conversación con nuestro mundo" (Mesa, 2013, p. 1).

A su vez, se estructuró una encuesta de 12 preguntas -sobre el logosímbolo, su impacto y el análisis sobre si Ecopetrol S.A. estaba inmerso en greenwashing - a profesionales de diversas disciplinas - Antropología, Artes, Biología marina, Diseño gráfico, Ecología, Publicidad y Sociología. Para lo que nos compete, fue destacable el concepto del artista y experto en estética, quien sostuvo que desde el punto de vista iconográfico, la iguana, como símbolo, puede relacionarse figurativamente a un camaleón (camuflaje, cambio, rápida respuesta), lo que afirma las ideas de adaptación, seguridad y armonía entre el medio ambiente y la actividad petrolera. 
Gráficamente, por una parte, cumple a cabalidad con los cuatro elementos mencionados al inicio: el tipo de tipografía, el color, el tamaño y el equilibrio, lo que resulta en un logosímbolo bien logrado, con equilibrio en el manejo de las cajas de texto - pues son completamente simétricas. La gama cromática, que va desde el amarillo hasta el verde oscuro, genera armonía; y la iguana, con su plácida pose, irradia tranquilidad. Por otra parte, se advierte que los conceptos de juventud y novedad son evidentes gracias a la tipografía en movimiento y los trazos caricaturescos de la iguana. Todos los elementos que la empresa quiso exaltar están presentes en el logosímbolo, el cual denota una personalidad fresca con la sensación de dirigirse con firmeza hacia el futuro. De ahí el mensaje: "energía para el futuro".

\section{Impacto del logosímbolo 2003}

Cuando el logosímbolo se adoptó en 2003, desde el punto de vista medioambiental el mundo empresarial atravesaba momentos de incertidumbre. A las entidades se les empezaba a exigir estándares de cumplimiento bastante estrictos, pues se tenían soportes científicos que involucraban a las actividades petroleras como responsables del $25 \%$ de los gases de efecto de invernadero (GEF) que causan el calentamiento global y el cambio climático.

Los primeros cuatro logosímbolos fueron nacionalistas y estuvieron inspirados en el tunjo precolombino; sin embargo, no cumplían con los requerimientos exigibles a la nueva empresa, por lo que renovar la imagen corporativa era urgente y evidente - máxime si el mundo, debido a los desequilibrios ambientales, impelía a un compromiso ético, de responsabilidad y de transformación empresarial. Ese imaginario del Estado asociado con Ecopetrol era un concepto desgastado, anacrónico y proclive a la corrupción -concepción generalizada en la sociedad colombiana respecto a los asuntos y empresas del Estado-, por lo que fue hábilmente remplazado por el de una empresa mixta, ágil y dinámica, que manifestaba estar comprometida con las obligaciones medioambientales globales y nacionales, ya que en caso contrario podría ser cuestionada social, económica y financieramente.

Consolidado el logosímbolo, el 23 de septiembre de 2007, Ecopetrol S.A. decidió emitir acciones para libre adquisición, y al cabo del primer minuto de operaciones en la Bolsa, estas se suspendieron porque las apuestas de los inversionistas fueron excesivas. En total, 495 mil colombianos del 95\% de los municipios del país compraron acciones, cifra que batió varios récords. Este acontecimiento quedó registrado en la historia del mercado accionario colombiano como "el efecto iguana" (Ecopetrol, 2012; Mesa, 2013, p. 1; Ultrabursátiles, 2013). El éxito incrementó, el 18 de septiembre del 2008, la empresa ingresó a la bolsa de Nueva York con bonos de deuda pública. A diciembre de 2017 alcanzó a tener en el mercado 41.116.688.048 acciones (Ecopetrol, 2017 p. 90), lo que impactó el mercado internacional y nacional.

Con el fin de tener una percepción a partir de un público informado, a estudiantes universitarios de Biología se les presentaron los aspectos positivos y negativos de la actividad petrolera, su relación con los problemas ambientales, y el concepto de greenwashing y de publicidad engañosa. Adicionalmente, una diseñadora gráfica analizó el contenido y significado del nuevo logosímbolo de Ecopetrol S.A. Con este panorama, al cabo de cuatro sesiones de trabajo de tres horas cada una, se implementó una encuesta con seis preguntas para conocer la percepción que tenían del logosímbolo. 39 de los 40 estudiantes conceptuaron que el logosímbolo no correspondía con la responsabilidad que se le podía imputar a las actividades petroleras, y solamente uno (1) -que familiarmente era accionista de la empresa- estuvo de acuerdo con el logosímbolo y su significado.

A su vez, los resultados de la encuesta a Omar Rincón, artista; a Ricardo Toledo, artista teórico; a Verónica Trimiño, antropóloga; a Sebastián Cuéllar, sociólogo; a un ecólogo -que solicitó reserva de su nombre -; a César Echenique, publicista; y a Wilfrido Solano Trujillo, biólogo marino, evidenciaron que todos coincidieron en el éxito del logosímbolo, y en que no había coherencia entre lo que se presentaba e intentaba 
comunicar, y sus impactos ambientales. En mayor o menor grado, todos coincidieron en que se incurría en publicidad engañosa, verderización y greenwashing.

\section{Conclusiones}

Respecto a los primeros cuatro logosímbolos de Ecopetrol, el último de 2003 abandonó el mensaje nacionalista y pasó a tener referentes ambientalistas, lo que acentuó la idea de que la actividad petrolera es "limpia". De igual manera, le comunicó a la sociedad y a los potenciales socios que estaba abierta a todos los colombianos, y que como era ambientalmente responsable, tendría aceptación en los mercados internacionales, lo que le abrió el camino hacia la fluidez financiera y crediticia. Apelando a las necesidades energéticas del país, impulsó la creencia colectiva de que desarrollo significa más energía -con origen en el petróleo-, por medio del mensaje "energía para el futuro", haciendo caso omiso a que todos los estudios reconocen que el petróleo ha llegado a su cénit de extracción, lo que hace casi que obligatorio empezar a utilizar las energías renovables, por lo que "debemos dejar al petróleo antes de que el petróleo nos deje a nosotros" (Brown, 2009, p. 48).

Publicitaria, comunicativa y económicamente, el impacto del nuevo logosímbolo fue evidente, de ahí el efecto "iguana". Gracias al branding, que fue inspirador, persuasivo, convincente y seductor, logró una identidad e imagen corporativa exitosa, y un valor de marca representado en su valor de mercado, logrando así posicionarse financieramente, a escala nacional e internacional.

Desde la óptica de la publicidad engañosa, verderización y greenwashing, Ecopetrol S.A. incurre en cinco de los siete pecados de este último concepto: (1) el pecado del precio oculto: la actividad petrolera no es "verde" en sí misma, sino por las acciones que promueve en pro del medio ambiente; (2) parcialmente puede ser aplicable el pecado de la falta de prueba, pues sus actividades medioambientales y productivas se encuentran certificadas como limpias y en cumplimiento de los estándares ambientales; (3) el pecado de la ambigüedad, ya que los mensajes publicitarios son generales y poco concretos; (4) el pecado de idolatrar falsas etiquetas, ya que el logosímbolo acude a expresiones como "Eco", a la iguana y a ciertos colores alusivos a buenas conductas ambientales; y (5) el pecado de mentir, pues los barriles no son limpios, ni en su extracción, ni en su proceso ni mucho menos en su utilización (Greenpeace, 2012; Alejos, 2013). Así lo ratificaron los siete expertos y los 39 de los 40 estudiantes universitarios consultados.

\section{Referencias}

Aaker, D. (1996). Measuring brand equity across products and markets. California Management Review, 38(3), 102-120.

Alejos, C. (2013). Greenwashing: ser verde o parecerlo. Recuperado de https://media.iese.edu/research/pdfs/ST-032 8.pdf

Ballestas, L. (2009). Las formas esquemáticas del diseño precolombino de Colombia: relaciones formales y conceptuales de la gráfica en el contexto cultural colombiano (tesis de doctorado). Universidad Complutense de Madrid, Madrid, España.

Balmer, J. (2008). Identity based views of the corporation. Insights from corporate identity, organizational identity, social identity, visual identity, corporate brand identity and corporate image. European Journal of Marketing, 42(9/10), 879-906.

Brown, L. (2009). Plan B 4.0: Mobilizing to save civilization. Nueva York: W.W. Norton \& Company.

Bucheli, M. (2010). Major trends in the historiography of the Latin American Oil Industry. Business History Review, 84(2), 339-362. Doi: 10.1017/S0007680500002646. Recuperado: abril 25 de 2018.

Caldevilla, D. (2010). La cara interna de la comunicación en la empresa. Madrid: Vision Net. 
Castrillón, M. (1961). Historial de las banderas y escudos nacionales. Bogotá: Revista de las Fuerzas Armadas de Colombia.

Chajet, C., y Shachtman, T. (1998). Image by design: from Corporate vision to business reality. Nueva York: McGrawHill.

Christensen, L., y Askegaard, S. (2001). Corporate identity and corporate image revisited: A semiotic perspective. European Journal of Marketing, 35(3/4), 292-315.

Constela-Umaña, A. (1995). Sobre el estudio diacrónico de las lenguas chibchenses y su contribución al conocimiento del pasado de sus hablantes. Boletin del Museo del Oro, (38-39), 13-56.

Corcho Tróchez, G. (2013). De las 10 petroleras más grandes, cinco tuvieron bajas en ingresos en 2013. La República. Recuperado de https://www.larepublica.co/empresas/de-las-10-petroleras-mas-grandes-cinco-tuvieron-bajas-e n-ingresos-en-2013-2119426.

Currás, R. (2010). Identidad e imagen corporativas: Revisión conceptual e interrelación. Universitat de València. Teoría $y$ Praxis, (7), 9-34.

De La Pedraja, T. R. (1993). Petróleo, electricidad, carbón y politica en Colombia. Bogotá: El Áncora.

Eco, U. (2010). La historia de la belleza. Madrid: Editorial De bolsillo.

Ecopetrol. (2011). El petróleo en Colombia 60 años. Ecopetrol. Bogotá: Villegas.

Ecopetrol. (2012, junio). Evento analistas e inversionistas (resumen de presentaciones). Recuperado de https://www. ecopetrol.com.co/wps/portal/es/ecopetrol-web/relacion-inversionistas/

Ecopetrol. (2017). Reporte integrado de gestión sostenible 2017. Bogotá: Beta Impresores SAS.

Forero, M. F., y Duque, E. J. (2014). Evolución y caracterización de los modelos de brand equity. Suma de Negocios, 5(12), 158-168.

Greenpeace. (2012). Greenwash+20. Cómo las grandes empresas se interponen en el camino hacia el desarrollo sostenible. Recuperado de https://archivo-es.greenpeace.org/espana/Global/espana/report/generales/gp_res_ejecutivo_g reenwash20.pdf

Healey, M. (2008). What is branding? Mies, Suiza: Roto Visión.

Hoyos, R. (2013). Plan de marketing: diseño, implementación y control. Bogotá: Ecoe.

Klein, N. (2001). No logo: El Poder de las marcas. Barcelona: Paidós.

Kotler, P., y Armstrong, G. (2008). Fundamentos de marketing (8.a ed.). Nueva Jersey: Pearson-Prentice Hall.

Kuhn, T. S. (2006). La estructura de las revoluciones cientificas (trad. C. Solís Santos) (3.a ed.). México D.F.: Fondo de Cultura Económica.

Lury, C. (2004). Brands. The logos of the global economy. Londres: Routledge.

Martínez, M. E. (2011). El branding, la sustentabilidad y el compromiso social del diseño. Cuando ser es más importante que parecer. Revista del Centro de Investigación, 9(35), 11-17.

Mesa, M. M. (2013). Nuestra iguana sigue creciendo. Corporate. Recuperado de http://corporate.la/nuestra-iguana-s igue-creciendo/

Ministerio de Minas y Energía. (2003, 26 de junio). Por el cual se escinde la Empresa Colombiana de Petróleos, Ecopetrol, se modifica su estructura orgánica y se crean la Agencia Nacional de Hidrocarburos y la sociedad Promotora de Energía de Colombia S. A. [Decreto 1760 de 2003]. DO: 45.230. Recuperado de http://www.se cretariasenado.gov.co/senado/basedoc/decreto_1760_2003.html

Montaña-Cuéllar, D. (1977). Colombia pais formal y pais real. Bogotá: Latina.

Olins, W. (2004). On brand. Nueva York: Thames \& Hudson.

Philip, G. (1982). Oil and politics in Latin America. Cambridge: Cambridge University Press.

Poiesz, T.B.C. (1988). The image concept: Its place in consumer psychology and its potential for other psychological areas. Ponencia presentada en XXIV International Congress of Psychology, Sidney.

Pol, A. (2005). Secretos de marcas, logotipos y avisos publicitarios: simbolismo gráfico, espacial y cromático. Buenos Aires: Dunken. 
Ricupero, S. (2007). Diseño gráfico en el aula: guia de trabajos prácticos. Madrid: Nobuko.

Sánchez, A. (1992). Invitación a la estética. México D.F.: Grijalbo.

Silva, B. Y. (2011). Reseña de "¿Qué es el branding" de Healey, M. Revista del Centro de Investigación, 9(35), 43-44.

Ultrabursátiles S. A. (2013). Mercado bursátil colombiano. Ecopetrol 2013. Recuperado de https://mercadobursatilco lombianofs.blogspot.com/2013/07/ecopetrol-2013.html

Van Riel, C. (1995). Comunicación corporativa. Madrid: Prentice Hall.

Vega, R., Núñez, L. A., y Pereira, A. (2009). Petróleo y protesta obrera: la USO y los trabajadores petroleros en Colombia (1923-2008). Bogotá: Corporación Aury Sará Marrugo.

Villegas, J. (1976). Petróleo colombiano ganancia gringa. Bogotá: Ediciones Peñaloza y Cía. Ltda.

Zanon, D. (2007). Introducción al diseño editorial. Madrid: Visión Net.

Notas

1 En las ciencias sociales, paradigma se describe como el conjunto de experiencias, creencias y valores que determinan la forma en la cual el individuo ve e interpreta la realidad, su realidad; y la forma en que responden a esa percepción. Es un patrón o modelo de conducta heredada o aprendida. $\mathrm{O}$ "un modelo o patrón aceptado, un arquetipo de investigación que tiene vigencia por un determinado tiempo; esta vigencia está dada, en primer lugar, por su poder o capacidad para resolver o solucionar problemas dentro del campo de la investigación científica; y en segundo lugar, está dada gracias a la lucidez de pensamiento con la cual se logra visualizar de manera nueva y creativa los diferentes interrogantes que se presentaban oscuros y confusos para el anterior paradigma de pensamiento" (Kuhn, 2006, pp. 71-78).

2 Universalmente las investigaciones y estudios realizados desde la psicología, la sociología, la grafología, la semiología y la antropología, evidencian la existencia de ciertas ideas apriorísticas establecidas de modo inconsciente, denominadas arquetipos. Es por ello que en cada época existe una especie de cosmovisión gráfica, esto es un conjunto de pautas o parámetros de "lectura" de las formas, que modela la percepción colectiva.

3 La defensa de los recursos naturales en América Latina y el Caribe en la década de los 50's a 60's, fue una postura ideológica de los movimientos de izquierda, en tiempos cuando las dictaduras eran el común denominador político de la región.

* Artículo de investigación científica.

\section{Licencia Creative Commons CC BY 4.0}

Cómo citar este artículo: Gutiérrez Gutiérrez, M. M. (2019). El logo, el branding y su influencia en la marca. Caso de estudio: el logosímbolo de Ecopetrol 2003. Signo y Pensamiento, 38(75). https://doi.org/10.1114 4/Javeriana.syp38-75.lbim 